\title{
Identificación de estilos de aprendizaje para la adaptación de un ambiente eLearning
}

\author{
Identification of learning styles for the adaptation of an eLearning environment
}

\author{
Yasunari del Valle Ramírez León ${ }^{1}$ \\ https://Orcid.Org/0000-0002-4309-6976 \\ Universidad Simón Bolivar, Venezuela
}

Recibido: 17-04-2019

Aceptado: 08-08-2019

\section{Cita Recomendada}

Ramírez, Y. (2019). Identificación de estilos de aprendizaje para la adaptación de un ambiente eLearning. Hamut'ay, 6(2), 126-140.

http://dx.doi.org/10.21503/hamu.v6i2.1779

\section{ReSUMEN}

Este documento trata de exponer en grandes líneas, una aproximación entre el eLearning y los estilos de aprendizaje. El objetivo fue identificar los estilos de aprendizaje para la adaptación de un ambiente eLearning. El marco metodológico adoptó el paradigma positivista, con un diseño no experimental descriptivo, transversal. Como resultado, se determinó que los 257 alumnos del programa eLearning Afectivo e Inclusivo: Principios y Estrategias, se orientan hacia el nivel equilibrado en las cuatro dimensiones de estilo de aprendizaje del modelo de Felder. En este contexto, también se evaluó la forma en que pudieran combinarse estos estilos, siendo la combinación predominante Activo-Sensitivo, SecuencialVisual, en un $45.66 \%$ de la población. Asimismo, pudo dilucidarse que en los ambientes eLearning basados en la plataforma Moodle, es posible adaptar la didáctica con base en la selección y articulación de material de instrucción compilado de diversas fuentes y en la definición de estrategias didácticas haciendo uso de las herramientas de colaboración de las cuales dispone el LMS (Learning Management Systems). Se concluye que la adaptación del diseño didáctico puede realizarse a través de las fases de detección de los estilos de aprendizaje de los discentes y la combinación de los mismos, selección de las estrategias didácticas, implementación de las estrategias a través de los recursos disponibles en Moodle y presentación de la interfaz según las estrategias y recursos seleccionados.

Palabras clave: estilo de aprendizaje, didáctica, eLearning, adaptatividad.

\section{Abstract}

This document is aimed to outline an approximation between eLearning and learning styles. The objective was to identify learning styles for the adaptation of an eLearning environment. The me-

${ }^{1}$ Auxiliar Docente V adscrita al Departamento de Tecnología Industrial de la Universidad Simón Bolívar, Venezuela. Dra. en Ciencias de la Educación por la Universidad de Granada, España. Profesora en la especialidad de Electrónica Universidad Pedagógica Experimental Libertador y Telemática por la Universidad Politécnica Territorial del Estado de Aragua,Venezuela. E-mail: ydelvramirez@usb.ve 
thodological framework adopted the positivist paradigm, with a descriptive, non-experimental, cross-sectional design. As a result, it was determined that the 257 students of the Affective and Inclusive eLearning program: Principles and Strategies, are oriented towards the balanced level in the four dimensions of the learning styles according to the Felder model. In this context, the way in which these styles could be combined was also evaluated, being predominant the Active-Sensitive, Sequential-Visual combination in a $45.66 \%$ of the population. Likewise, it could be elucidated that in the eLearning environments based on the Moodle platform, it is possible to adapt the didactics based on the selection and articulation of instructional material compiled from various sources and in the definition of didactic strategies using the collaboration tools available in the LMS (Learning Management Systems). It is concluded that the adaptation of the didactic design can be carried out through the detection phases of the learning styles of the students and their combination, selection of teaching strategies, implementation of strategies through the resources available in Moodle and the presentation of the interface according to the selected strategies and resources.

Keywords: learning style, didactic, eLearning, adaptivity.

\section{INTRODUCCIÓN}

La inserción de las tecnologías como herramienta de apoyo para los procesos formativos on line, han permitido flexibilizar el acceso a los diversos sistemas educativos al no requerir estar en un espacio físico, ni estar limitado a un tiempo específico de enseñanza; sino por el contrario, brindar una amplia gama de programas formativos y mayor accesibilidad a los mismos, desde la distancia.

Desde esta perspectiva la comunidad académica e investigativa emerge como un modelo de enseñanza que promueve y orienta la optimización de la educación en los entornos virtuales, en la que toma en cuenta algunas características propias del estudiante, entre las que se encuentra los estilos de aprendizaje. Así, surgen estudios como los de Paredes \& Rodríguez (2006), Iglesias, Ortiz-Repiso \& Picazo, (2006), Paredes (2008), Paredes \& Rodríguez, (2004), Pesantez, (2008) y Vélez (2009); quienes han coincidido en el análisis de las formas de aprender desde la propuesta Felder \& Soloman, como base para adaptar nuevas tecnologías al proceso de enseñanza. Posteriormente, los trabajos realizados por Antelm, Cacheiro-González \& Gil-López, (2015) y Gutiérrez, (2018), develan la importancia de detectar las formas de aprender que tienen los alumnos con los que se trabaja, ya que es un factor determinante para adaptar la forma de enseñar a los estilos de aprender y de este modo poder contribuir a mejorar el rendimiento académico de los discentes.

Fue de interés para la presente investigación, efectuar la revisión de los estudios realizados por Porras (2017), Macías-Romero (2015) y Gutiérrez, (2018); por cuanto sus aportes confirman la viabilidad de adaptar estrategias didácticas dentro de un entorno virtual eLearning basado en Moodle y de modelar un ambiente educativo virtual, empleando los módulos disponibles en el citado LMS, de acuerdo con las formas de aprender, definidas a través de las dimensiones que abarca el modelo de Felder \& Soloman.

En este contexto, el objetivo de este estudio se centra en el diseño didáctico adaptado a las formas de aprender referidas a entornos virtuales, por lo que surge la siguiente interrogante: ¿De qué manera el diseño didáctico se puede adaptar a las formas de aprender de los estudiantes en un ambiente eLearning? Y se definió como objetivo general de esta investigación: identificar los estilos de aprendizaje para la adaptación de un ambiente eLearning teniendo como objetivos específicos: i. Realizar una revisión de la literatura acerca de los estilos de aprendizaje y de los conceptos re- 
lacionados con el diseño didáctico en entornos educativos virtuales basados en LMS. ii. Conocer las formas de aprender en los estudiantes del programa eLearning Afectivo e Inclusivo: Principios y Estrategias. iii. Relacionar las características adaptables del LMS Moodle con las formas de aprender de los discentes registrados en la plataforma de teleformación del Grupo TEIS, que soporta el programa formativo base de este estudio. iv. Proponer una metodología de adecuación del esquema didáctico a las formas de aprender de los estudiantes del programa eLearning Afectivo e Inclusivo: Principios y Estrategias.

\section{Estilos de Aprendizaje}

Las formas de aprender han sido investigadas desde los ańos sesenta, y son múltiples los enfoques y conceptualizaciones que han surgido al respecto. El término "estilos de aprendizaje" según Felder, hace referencia a "las preferencias y características en cuanto a la forma en que un estudiante percibe y procesa la información” p.19. A este respecto, Alonso, Duque \& Correa (2013), concluyen que no existe una unívoca manera de aprender, por tanto; no existe una única tipología de formas de aprender. En relación a la variedad de modelos de estilo de aprendizaje existentes, los citados autores acotan que éstos se pueden clasificar en categorías con base en los enfoques en cuanto a: forma de construir el conocimiento por parte del educando, la experiencia, las vías más expeditas de captación de información del discente, las estrategias instruccionales, la interacción con otras personas, la dicotomía cerebral (a nivel de hemisferios) y la personalidad. Asimismo, Alonso, Duque \& Correa (2013), hacen referencia a las teorías de Felder \& Silverman (1988), Felder \& Soloman (1996) y Kolb (1973), destacando la relación que tienen en cuanto a la dimensión común de la percepción. Adicionalmente, recalcan la consistencia en las distintas versiones del modelo de Felder, identificándolo como un modelo basado en los canales de percepción de información. En cuanto a la percepción, Vilatuña et al., (2012), consideran que ésta es fundamental en la construcción del conocimiento, puesto que contribuye al desarrollo de las formas cognitivas superiores. Con base en este planteamiento, se se- lecciona la categorización de formas de aprender de Felder \& Soloman, a efectos del desarrollo de esta investigación.

\section{El modelo de Felder \& Soloman}

En cuanto a este modelo de estilos de aprendizaje existen diferentes posturas. Así, se tiene la de Macías-Romero (2015), quien subraya que este modelo describe al aprendizaje como un proceso en el cual los sentidos reciben la información y, además, en el que los estudiantes procesan la información de interés y desechan aquella que no consideran relevante. Ventura, Moscoloni \& Gagliardi, (2012), agregan que la formulación del modelo de Felder retoma las dimensiones de percepción y procesamiento propuestas por Kolb. Cruz, (2017) por su parte, coincide con la visión de Alonso, Duque \& Correa (2013), en cuanto a que este modelo define la forma en la que el estudiante distingue y asimila la información.

Tomando como base las inferencias de los trabajos citados anteriormente se escoge para este estudio el modelo de Felder \& Soloman, por cuanto el diseño didáctico -a consideración de la autora-, se relaciona en este contexto directamente con el proceso de elección, alineación y despliegue de distintos objetos de aprendizaje en la interfaz del ambiente eLearning y esto, a su vez, implica definir el cómo se va a entregar la información a los discentes para que la perciban de la mejor manera posible y adicionalmente a ello, Felder tiene una forma muy particular de describir las dimensiones que incluye en su modelo.

Diseño didáctico en ambientes virtuales de aprendizaje

Según Renés (2018), la heterogeneidad educativa puede verse favorecida cuando se incorporan las formas de aprender de los discentes en la práctica docente. Humanante, García-Peñalvo \& Conde (2015), concluyen que, en los ambientes virtuales, las secuencias didácticas deben estar diseñadas para promover el uso y desarrollo de las funcionalidades de los LMS y así optimizar el uso de las nuevas tecnologías aplicables al ámbito educativo. Según Amaro (2011), el enfoque didáctico con- 
de este estudio, por cuanto servirá de base para definir la adaptabilidad del diseño pedagógico, integrando los recursos del LMS Moodle.

Adaptabilidad de Moodle a estilos de aprendizaje.

Según los hallazgos de Paramythis \& Loidl (2004), un entorno educativo se considera adaptable si cumple cuatro características, las cuales se explican en la Figura 2.

Figura 2.

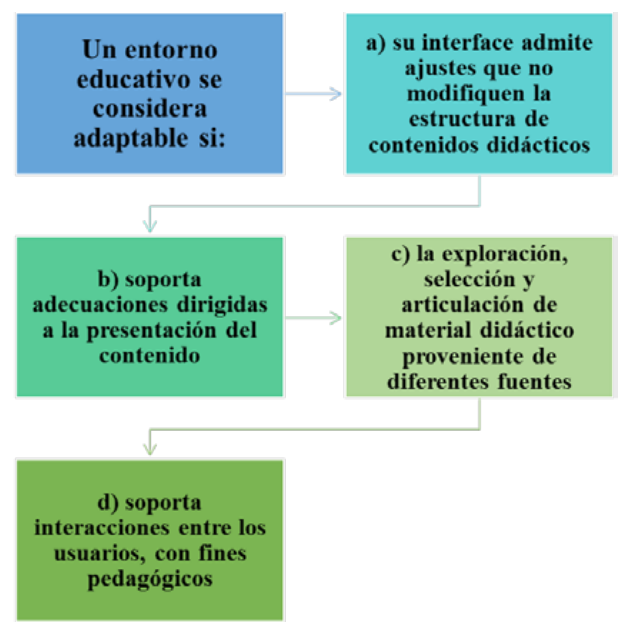

Características de un entorno educativo adaptable, Paramythis \& Loidl (2004).
Graf (2007), estudió nueve entornos de aprendizaje diferentes incorporando adaptabilidad. Observó que la plataforma Moodle mostró los mejores resultados.

En cuanto a la interacción pedagógica, el estudio de Revelo, Collazos \& Jiménez (2018) destaca que el trabajo colaborativo se está perfilando en los actuales momentos como una estrategia que tiene validez y pertinencia a los fines de promover el aprendizaje a través de la interacción de los alumnos, que no deja de lado la responsabilidad individual de cada miembro que participa (autogestión del conocimiento) y considera la heterogeneidad en la formación de los grupos. Este aspecto se considera importante, pues se puede inferir que es posible a través de los recursos y las actividades del LMS Moodle, construir grupos heterogéneos (considerando combinaciones de modos de aprender) e incluir en el diseño de la interfaz, actividades que involucran interacción con fines pedagógicos.

Despotovic \& Zrakic (2012), realizaron un análisis de los diferentes recursos de la plataforma Moodle y su utilidad según cada tipo de aprendiz, desde la perspectiva de las formas de aprender planteadas por Felder \& Soloman. Sugirieron un diseño didáctico haciendo uso de los recursos que se plantean en la Tabla 1.

Tabla 1.

Recursos Moodle analizados según cada estilo de aprendizaje

\begin{tabular}{|c|c|c|c|c|c|c|c|c|}
\hline & \multicolumn{8}{|c|}{ Actividades Moodle } \\
\hline & Foro & Chat & Glosario & Taller & Encuesta & Opción & Lección & $\begin{array}{l}\text { Método de } \\
\text { Colaboración }\end{array}$ \\
\hline Activo & $\begin{array}{l}\text { Problemas } \\
\text { concretos }\end{array}$ & Sí & $\begin{array}{l}\text { Muchos } \\
\text { términos }\end{array}$ & Experimentos & No & Sí & $\begin{array}{l}\text { Ejemplos de } \\
\text { problemas }\end{array}$ & Presencial \\
\hline Reflexivo & Temas reflexivos & No & Conceptos & $\begin{array}{l}\text { Temas explo- } \\
\text { rados }\end{array}$ & Sí & Sí & $\begin{array}{l}\text { Temas pro- } \\
\text { porcionados }\end{array}$ & Email \\
\hline Visual & No & No & No & Sí & Sí & Sí & Ilustración & Combinado \\
\hline Verbal & Sí & Sí & Sí & Sí & Sí & Sí & $\begin{array}{l}\text { Escrito, } \\
\text { Multimedia }\end{array}$ & Combinado \\
\hline Secuencial & Sí & Frecuente & Sí & Sí & No & Sí & Sí & Combinado \\
\hline Global & Temas Globales & No & No & Sí & Sí & Poco & Poco & Combinado \\
\hline Sensitivo & $\begin{array}{l}\text { Hechos, ejem- } \\
\text { plos }\end{array}$ & Sí & Sí & $\begin{array}{l}\text { Ejemplos } \\
\text { Prácticos }\end{array}$ & Sí & Sí & $\begin{array}{l}\text { Hechos, } \\
\text { Algoritmos }\end{array}$ & Combinado \\
\hline Intuitivo & $\begin{array}{l}\text { Temas abstrac- } \\
\text { tos }\end{array}$ & No & No & & Sí & No & Poco & Combinado \\
\hline
\end{tabular}

Fuente: Despotovic \& Zrakic (2012, p.328). 
Después de haber estudiado la didáctica para estilos de aprendizaje Gamero, (2014), aconseja que para los estilos activo y visual es recomendable emplear técnicas didácticas basadas en resolución de problemas, en el uso del correo electrónico y búsquedas en fuentes externas de Internet; las cuales se podrán emplear como complemento a las actividades dentro del ambiente eLearning.

\section{Materiales Y Métodos}

\section{Participantes}

Se aplicó la técnica de muestreo por conveniencia (Otzen \& Manterola, 2017), siendo la muestra el total de la población de estudiantes $(n=257)$ matriculados en el programa eLearning Afectivo e Inclusivo: Principios y Estrategias; y, asimismo, registrados en el LMS que soporta al citado programa formativo, propiedad del Grupo TEIS HUM-848, vinculado a la Universidad de Granada. La Tabla 2, muestra las características generales de edad, género y número de los participantes.

Tabla 2

Distribución en edad, género y número de la muestra

\begin{tabular}{lll}
\hline Edad & $\begin{array}{l}\text { El } 54.1 \% \text { de los participantes en el pro- } \\
\text { grama, se ubican en el rango de edad de } \\
\text { 23-45 años. }\end{array}$ \\
\hline Género & Cantidad & $\%$ \\
\hline Femenino & 193 & 75.09 \\
Masculino & 64 & 24.90 \\
\hline Total & 257 & 100.00 \\
\hline
\end{tabular}

Fuente: Elaboración propia (2018).

\section{Instrumento}

A efectos de la presente investigación, se empleó el instrumento desarrollado por Felder \& Soloman, cuya estructura se resume en la Tabla 3. Como puede observarse, este cuestionario está constituido por 44 ítems, divididos en 4 grupos de 11 preguntas cada uno. Cada grupo de ítems representa a cada dimensión de estilo de aprendizaje definida en el modelo de Felder. Los ítems son de opción dicotómica (a y b), de respuesta selectiva y tipo escala.
El cuestionario califica a cada estilo en tres niveles de preferencia, a saber: Fuerte (9-11), Moderado (5-7) y Equilibrado (1-3). El perfil del discente se obtiene totalizando tanto el número opciones seleccionadas "a", como el número de opciones seleccionadas "b" para cada grupo de ítems. Luego, al valor mayor, se le sustrae el menor. El resultado se indica, conjuntamente con la letra relacionada más veces. El número indica el nivel y la letra, la dimensión preferida (la "a" al extremo izquierdo y la "b", al extremo derecho de la escala). De este modo, se determina cómo el discente adquiere, percibe, procesa y comprende la información.

Tabla 3

Descripción del Test de Felder

\begin{tabular}{|c|c|c|c|}
\hline Dimensiones & $\begin{array}{l}\text { Pregun- } \\
\text { tas/di- } \\
\text { mensión }\end{array}$ & Tipo & Ítems \\
\hline Activo/Reflexivo & 11 & $\begin{array}{l}\text { Dicotómico } \\
\text { (a ó b) }\end{array}$ & $\begin{array}{l}1,5,9,13,17 \\
21,25,29,33 \\
37,41\end{array}$ \\
\hline $\begin{array}{l}\text { Sensitivo/Intui- } \\
\text { tivo }\end{array}$ & 11 & $\begin{array}{l}\text { Dicotómico } \\
\text { (a ó b) }\end{array}$ & $\begin{array}{l}2,6,10,14,18 \\
22,26,30,34, \\
38,42\end{array}$ \\
\hline Visual/Verbal & 11 & $\begin{array}{l}\text { Dicotómico } \\
\text { (a ó b) }\end{array}$ & $\begin{array}{l}3,7,11,15,19 \\
23,27,31,35 \\
39,43\end{array}$ \\
\hline $\begin{array}{l}\text { Secuencial/ } \\
\text { Global }\end{array}$ & 11 & $\begin{array}{l}\text { Dicotómico } \\
\text { (a ó b) }\end{array}$ & $\begin{array}{l}4,8,12,16,20 \\
24,28,32,36 \\
40,44\end{array}$ \\
\hline
\end{tabular}

Fuente: Adaptado de International Centre for Educator's Learning Styles, (2018).

Para llevar a cabo la recogida de datos a través del instrumento seleccionado, se ejecutaron una serie de adecuaciones conducentes a embeber el test en la interfaz del ambiente eLearning del programa eLearning Afectivo e Inclusivo: Principios y Estrategias; con el fin de que los alumnos pudieran completar el instrumento al ingresar por primera vez en la plataforma. Estas adecuaciones incluyeron entre otros aspectos, la traducción del idioma inglés al español, la publicación del instrumento traducido en un dominio web (como documento html) y el empleo de la url respectiva para embeber el instrumento en el frontend. Asimismo, se erigió una base de datos para registrar las respuestas al test una vez completado -a nivel de backend-, cuyo contenido se exportó posteriormente a fin de procesar las respuestas obtenidas. 
En relación a la confiabilidad del instrumento se realizó con el coeficiente del Alfa de Cronbach, que según Chavez (2007), es una técnica adecuada para obtener la confiabilidad de un instrumento, cuando se aplica una sola vez. Este autor indica que el referido coeficiente, puede alcanzar valores entre 0 y 1 . Valores cercanos o iguales a 0 , significan nula confiabilidad; mientras que los valores cercanos o iguales a 1 , representan la confiabilidad total. El valor del Alfa de Cronbach se puede obtener a través de herramientas informáticas orientadas al análisis de datos, como lo es SPSS de IBM, que, a los fines de este estudio, fue empleado en su versión 21. Los resultados de esta prueba se muestran en la Tabla 4.

Tabla 4

Alfa de cronbach obtenidos para el test de Felder.

\begin{tabular}{lc}
\hline Deficiencia cognitiva & $\mathrm{Cl}$ \\
\hline Activo/Reflexivo & 0.935 \\
Sensitivo/Intuitivo & 0.967 \\
Visual/Verbal & 0.857 \\
Secuencial/Global & 0.988 \\
\hline
\end{tabular}

Fuente: Elaboración propia (2018).

Según la Tabla 4, el instrumento se considera fiable, pues los valores obtenidos de la prueba luego de su aplicación, son mayores de 0.5 , que según Felder \& Spurlin (2005) se considera suficiente; a pesar de que el coeficiente más bajo (dimensión Visual/Verbal), en relación con el resto de las dimensiones no alcanza un valor de 0.9.

\section{Tipo y diseño}

Con base en lo descrito por Hernández, Fernández \& Baptista (2014), esta investigación adoptó al paradigma positivista.

El diseño fue descriptivo transversal, no experimental; orientado a detectar las características referidas a las formas de aprender de un grupo de estudiantes en específico y en función de las mismas, describir un método de adaptación del diseño didáctico a estas características; enmarcando el proceso dentro de un estudio de caso.

\section{Procedimiento}

Este estudio se estructuró en las cuatro etapas que se esquematizan en la Figura 3.

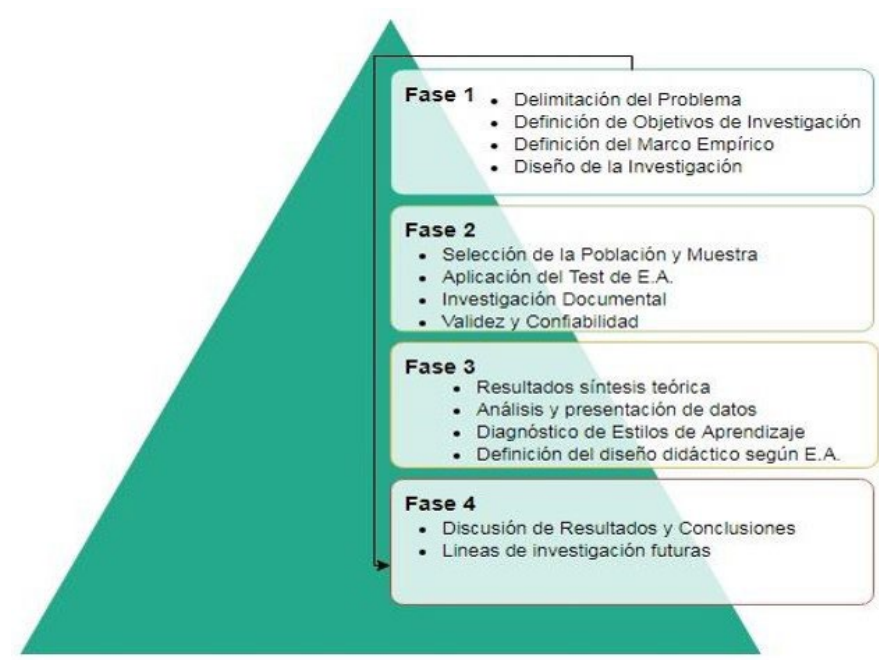

Figura 3.

Etapas de la investigación. Elaboración propia (2018).

\section{Confidencialidad}

A los fines de materializar esta investigación, se contó con el apoyo del Grupo de Investigación TEIS (Tecnología Educativa e Investigación Social) HUM-848, quien cedió los privilegios de administración de la plataforma Moodle que sirve de soporte al ambiente eLearning del programa formativo objeto del caso de estudio. En cuanto a la participación de los estudiantes, se les invitó por medio de correo electrónico a participar respondiendo el Test de Felder, remitido por el tutor principal del programa eLearning Afectivo e Inclusivo: Principios y Estrategias.

\section{Resultados}

Se presentan los resultados de este estudio, que pretenden responder a la interrogante planteada en las secciones preliminares de este documento.

\section{Estilos de Aprendizaje detectados por dimensión}

A los fines de realizar una interpretación adecuada de los datos, se empleó la escala utilizada por Felder \& Spurlin (2005) y Guanipa \& Mogollón (2006), quienes, en sus investigaciones, estiman 
tres niveles: fuerte, moderado y equilibrado.

Así, para la escala dicotómica Secuencial/Global, la Tabla 5 muestra los resultados; habiendo obtenido de manera general un $66.14 \%$ (170) de la población, se caracteriza por el nivel de equilibrio entre los dos estilos. De igual forma, se tiene que un $15.17 \%$ (39) se ubica en el nivel moderado del estilo Global y un $13.61 \%$ (35) se ubica en el nivel moderado Secuencial, respectivamente. En los niveles fuertes, predomina el estilo Secuencial, sobre el Global observándose un predominio del género femenino en todos los niveles.

\section{Tabla 5}

Dimensión Secuencial-Global * Género

\begin{tabular}{lrcc}
\hline \multirow{2}{*}{$\begin{array}{l}\text { Dimensión } \\
\text { Secuencial-Global }\end{array}$} & Femenino & Masculino & $\begin{array}{c}\text { Total } \\
\text { General }\end{array}$ \\
\cline { 2 - 4 } & 2.72 & 0.78 & 3.50 \\
\hline Fuerte Secuencial & 8.60 & 5.00 & 13.61 \\
Moderado Secuencial & 49.8 & 16.34 & 66.14 \\
Equilibrio & 13.23 & 1.94 & 15.17 \\
Moderado Global & 0.79 & 0.78 & 1.56 \\
Fuerte Global & 0.99 & & 100 \\
Secuencial/Global & 75.10 & 24.90 \\
\hline \multicolumn{1}{c}{ Total } & & \\
\hline
\end{tabular}

Fuente: Elaboración propia (2018).

Para la escala dicotómica Activo/Reflexivo, la Tabla 6 muestra que un $55.64 \%$ de la población (143), se inclina por el balance entre los dos estilos. Asimismo, se tiene que para los niveles moderado $29.18 \%$ (75) y fuerte $10.50 \%$ (27) existe una inclinación hacia el estilo Reflexivo, con predominio del género femenino en todos los niveles.

Tabla 6

Dimensión Activo-Reflexivo * Género

\begin{tabular}{lrcc}
\hline \multirow{2}{*}{$\begin{array}{l}\text { Dimensión } \\
\text { Activo-Reflexivo }\end{array}$} & Femenino & Masculino & $\begin{array}{c}\text { Total } \\
\text { General }\end{array}$ \\
\cline { 2 - 4 } & 0.38 & 0 & 0.38 \\
\hline Fuerte Activo & 2.33 & 1.95 & 4.28 \\
Moderado Activo & 42.41 & 13.23 & 55.64 \\
Equilibrio & 21.79 & 7.39 & 29.18 \\
Moderado Reflexivo & 8.17 & 2.33 & 10.50 \\
Fuerte Reflexivo & 0.99 & & \\
Secuencial/Global & 75.10 & 24.90 \\
\hline \multicolumn{1}{c}{ Total } & & 100 \\
\hline
\end{tabular}

Fuente: Elaboración propia (2018).
La distribución para la escala dicotómica Sensitivo/Intuitivo, se muestra en la Tabla 7. De manera general un $61.47 \%$ (158) presenta la preferencia del balance entre los dos estilos. Del mismo modo, se tiene que para el nivel moderado un $14.78 \%$ (38) se identifica como Sensitivo y al mismo tiempo otro $14.78 \%$ (38) como Intuitivo. En el nivel fuerte se tiene una tendencia hacia el estilo Intuitivo con un 6.61\% (17).

Tabla 7

Dimensión Sensitivo-Intuitivo * Género

\begin{tabular}{lccc}
\hline \multirow{2}{*}{$\begin{array}{l}\text { Dimensión } \\
\text { Sensitivo-Intuitivo }\end{array}$} & Femenino & Masculino & $\begin{array}{c}\text { Total } \\
\text { General }\end{array}$ \\
\cline { 2 - 4 } & 1.55 & 0.77 & 2.33 \\
\hline Fuerte Sensitivo & 11.67 & 3.11 & 14.78 \\
Moderado Sensitivo & 45.52 & 15.95 & 61.47 \\
Equilibrio & 10.50 & 4.28 & 14.78 \\
Moderado Intuitivo & 5.83 & 0.77 & 6.61 \\
Fuerte Global & 0.99 & & \\
Secuencial/Global & 75.10 & 24.90 & 100 \\
\hline \multicolumn{2}{c}{ Total } & & \\
\hline
\end{tabular}

Fuente: Elaboración propia (2018).

Al evaluar la distribución Visual/Verbal mostrada en la Tabla 8 , se tiene que de manera general se presenta un equilibrio entre los dos estilos en un 35\% (90). El nivel moderado se inclina en un $33.46 \%$ de la población (86) hacia el estilo Verbal. En el nivel fuerte, también predomina la preferencia hacia el estilo Verbal en un $21.40 \%$ (55) de la población objeto de estudio, con predominio del género femenino en todos los niveles.

Tabla 8

Dimensión Visual-Verbal * Género

\begin{tabular}{lrcc}
\hline \multirow{2}{*}{$\begin{array}{l}\text { Dimensión } \\
\text { Visual-Verbal }\end{array}$} & \multicolumn{3}{c}{ Género } \\
\cline { 2 - 4 } & Femenino & Masculino & $\begin{array}{c}\text { Total } \\
\text { General }\end{array}$ \\
\hline Fuerte Visual & 1.94 & 0.39 & 2.33 \\
Moderado Visual & 4.28 & 3.50 & 7.78 \\
Equilibrio & 28.01 & 7.00 & 35.01 \\
Moderado Verbal & 25.68 & 7.78 & 33.46 \\
Fuerte Verbal & 15.17 & 6.22 & 21.40 \\
Secuencial/Global & 0.99 & & \\
\hline \multicolumn{2}{c}{ Total } & 75.10 & 24.90 \\
\hline
\end{tabular}

Fuente: Elaboración propia (2018). 


\section{Combinaciones de Estilos de Aprendizaje}

Según los resultados presentados hasta este punto, se evidencia que, por dimensión, la población objeto de estudio se caracteriza por ser Global-Reflexivo-Intuitivo - Verbal. En este contexto, también se evaluó la forma en que pudieran combinarse estos estilos, contemplando la totalidad de las dimensiones en la población objeto de estudio. La Tabla 9 muestra los resultados obtenidos en relación a este tópico.

Tabla 9

Combinación de estilos de aprendizaje * Género.

\begin{tabular}{|c|c|c|c|}
\hline \multirow[b]{2}{*}{ Combinaciones $2^{4}$} & \multicolumn{3}{|c|}{ Género } \\
\hline & Femenino & Masculino & $\begin{array}{c}\text { Total } \\
\text { General }\end{array}$ \\
\hline $\begin{array}{l}\text { Activo-Intuitivo, } \\
\text { Global-Visual }\end{array}$ & 13.62 & 3.50 & 17.12 \\
\hline $\begin{array}{l}\text { Reflexivo-Sensitivo, } \\
\text { Secuencial-Verbal }\end{array}$ & 1.56 & 0.39 & 1.95 \\
\hline $\begin{array}{l}\text { Activo-Intuitivo, } \\
\text { Global-Verbal }\end{array}$ & 5.45 & 1.17 & 6.61 \\
\hline $\begin{array}{l}\text { Reflexivo-Sensitivo, } \\
\text { Secuencial-Visual }\end{array}$ & 5.45 & 0.00 & 5.45 \\
\hline $\begin{array}{l}\text { Activo-Intuitivo, } \\
\text { Secuencial-Visual }\end{array}$ & 7.39 & 2.72 & 10.12 \\
\hline $\begin{array}{l}\text { Reflexivo-Sensitivo, } \\
\text { Global-Verbal }\end{array}$ & 1.17 & 1.17 & 2.33 \\
\hline $\begin{array}{l}\text { Activo-Intuitivo, } \\
\text { Secuencial-Verbal }\end{array}$ & 1.17 & 0.00 & 1.17 \\
\hline $\begin{array}{l}\text { Reflexivo-Sensitivo, } \\
\text { Global-Visual }\end{array}$ & 1.17 & 1.17 & 2.33 \\
\hline $\begin{array}{l}\text { Activo-Sensitivo, } \\
\text { Global-Visual }\end{array}$ & 10.51 & 4.67 & 15.18 \\
\hline $\begin{array}{l}\text { Reflexivo-Intuitivo, } \\
\text { Secuencial-Verbal }\end{array}$ & 1.17 & 0.78 & 1.95 \\
\hline $\begin{array}{l}\text { Activo-Sensitivo, } \\
\text { Global-Verbal }\end{array}$ & 0.78 & 0.39 & 1.17 \\
\hline $\begin{array}{l}\text { Reflexivo-Intuitivo, } \\
\text { Secuencial-Visual }\end{array}$ & 1.17 & 1.56 & 2.72 \\
\hline $\begin{array}{l}\text { Activo-Sensitivo, } \\
\text { Secuencial-Visual }\end{array}$ & 15.95 & 4.67 & 20.62 \\
\hline $\begin{array}{l}\text { Reflexivo- Intuitivo, } \\
\text { Global-Verbal }\end{array}$ & 2.33 & 1.17 & 3.50 \\
\hline $\begin{array}{l}\text { Activo-Sensitivo, } \\
\text { Secuencial-Verbal }\end{array}$ & 2.72 & 0.78 & 3.50 \\
\hline $\begin{array}{l}\text { Reflexivo- Intuitivo, } \\
\text { Global-Visual }\end{array}$ & 3.50 & 0.78 & 4.28 \\
\hline Total & 75.10 & 24.9 & 100 \\
\hline
\end{tabular}

Fuente: Elaboración propia (2018).
Los resultados mostrados en la Tabla 9 revelan la preferencia de la combinación de estilos Activo-Sensitivo, Secuencial-Visual en la población objeto de estudio. La misma se ubica en un $20.62 \%$ (53), con predominio del género femenino. Esta combinación es si se quiere, complementaria a la obtenida al evaluar el estilo por dimensión en forma individual. La Figura 4, muestra gráficamente esta distribución.

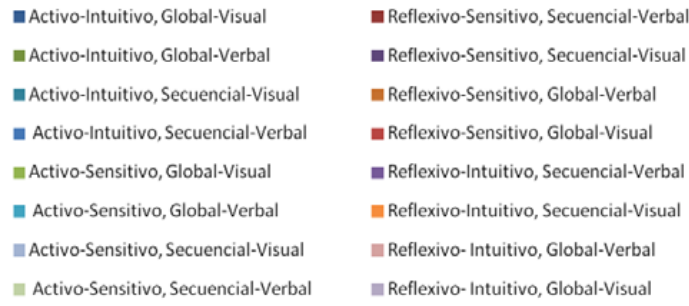

- Activo-Intuitivo, Global-Visual

activo-Intuitivo, Global-Verbal

activo-Intuitivo, Secuencial-Visual

- Activo-Intuitivo, Secuencial-Verbal

= Activo-Sensitivo, Global-Visual

n Activo-Sensitivo, Global-Verbal

॥ 1 Activo-Sensitivo, Secuencial-Visual

= Activo-Sensitivo, Secuencial-Verbal

- Reflexivo-Sensitivo, Secuencial-Verbal

- Reflexivo-Sensitivo, Secuencial-Visual

neflexivo-Sensitivo, Global-Verbal

- Reflexivo-Sensitivo, Global-Visual

= Reflexivo-Intuitivo, Secuencial-Verbal

= Reflexivo-Intuitivo, Secuencial-Visual

m Reflexivo-Intuitivo, Global-Verbal

m Reflexivo-Intuitivo, Global-Visual

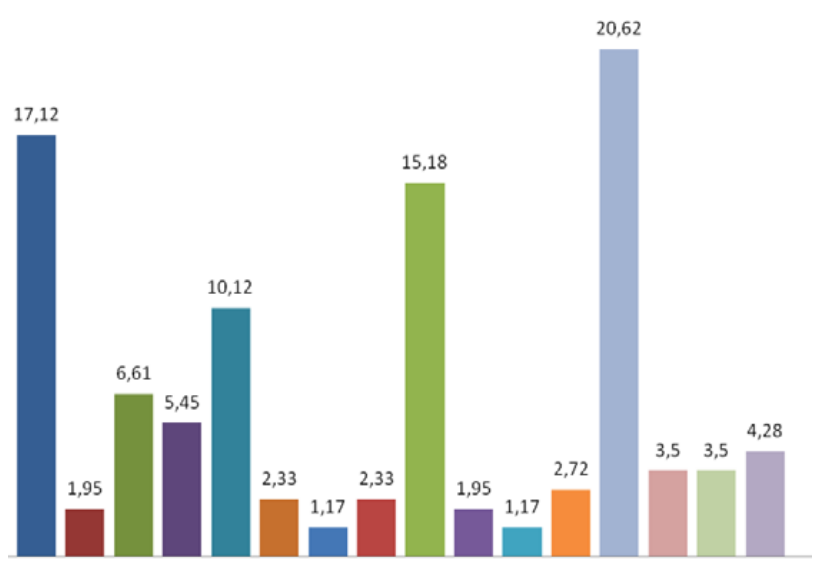

Figura 4.

Estilos combinados.

Elaboración propia (2018).

Atendiendo a lo indicado por Kanninen (2009) y Cruz Garzón (2017), se consideró también, esta combinación a los efectos de la prosecución del presente estudio.

Habiendo logrado los dos primeros objetivos específicos de esta investigación, se procedió a abordar el tercero sobre la base de la revisión documental efectuada. En tal sentido, para relacionar los estilos de aprendizaje preferidos con las estrategias didácticas, se asumieron los criterios que a continuación se enuncian:

- Se tomarán en cuenta para establecer la relación estrategias-estilos los recursos disponibles en la plataforma Moodle, en razón de que es el ambiente eLearning que soporta el programa formativo objeto de la presente investigación. 
- La relación estrategias-estilos, se establece sobre la base de lo planteado por Despotovic \& Zrakic (2012). Esto obedece a que la estructura genérica de Moodle, conserva la totalidad de los módulos referidos a las actividades, considerados por el citado autor.

- Se adoptó el análisis provisto en la investigación de Kanninen (2009); quien recomienda que el diseño didáctico considere la combinación preferida de estilos de aprendizaje, detectadas en el alumnado. En este caso, la combinación Activo-Sensitivo, Secuencial-Visual. Esta combinación también habrá de tenerse en cuenta a efectos de la inclusión de estrategias didácticas de tipo colaborativo, en atención a lo expresado por Angel (2015) y Revelo, Collazos \& Jiménez, (2018).

Procedimiento de adaptación del diseño didáctico a estilos de aprendizaje

A los fines de elaborar la propuesta objeto de esta investigación, se consideró los siguientes aspectos:

1. La plataforma que soporta el ambiente eLearning del Grupo TEIS, en el que se aloja el programa eLearning Afectivo e Inclusivo: Principios y Estrategias, es Moodle. Por lo tanto, se considera viable emplear las actividades y recursos disponibles en este LMS, con miras a proponer una versión adaptativa de la interfaz gráfica para los distintos tipos de estilos de aprendizaje definidos en el modelo de Felder \& Soloman.

2. Considerar las sugerencias de Despotovic \& Zrakic (2012), de manera que el diseño didáctico incluya los recursos que se plantean en la Tabla 1 de este documento en consonancia con la combinación de los estilos de aprendizaje predominante en los participantes del programa formativo referido y lo propuesto por Kanninen (2009).

3. Emplear, como guía para la adaptación del diseño didáctico, las instrucciones presentada por Graf (2007) con actividades y características de Moodle recomendadas para los diferentes estilos de aprendizaje. (Tabla 10).

4. Tomar en cuenta las recomendaciones efectuadas por Angel, (2015) y Revelo, Collazos
\& Jiménez, (2018), en relación a incluir en el diseño pedagógico actividades de trabajo colaborativo que beneficien el aprendizaje de discentes con distintas maneras de aprender, así como el empleo de herramientas externas al aula virtual que complementen y refuercen la comunicación síncrona y asíncrona de los participantes.

Tabla 10

Sugerencias de diseño para cada estilo de aprendizaje

\begin{tabular}{|c|c|c|}
\hline & Femenino & Masculino \\
\hline Activo & $\begin{array}{l}\text { Muchos ejercicios. Pruebas de } \\
\text { autoevaluación.Esquema antes } \\
\text { de introducir el contenido. }\end{array}$ & Ejemplos. \\
\hline Reflexivo & $\begin{array}{l}\text { Esquemas adicionales en } \\
\text { medio de los temas. Primero } \\
\text { la explicación del tema, luego } \\
\text { los ejemplos. Las conclusio- } \\
\text { nes se presentan de manera } \\
\text { evidente luego del contenido de } \\
\text { enseñanza. }\end{array}$ & $\begin{array}{l}\text { Ejercicio. } \\
\text { Pruebas de } \\
\text { auto evalua- } \\
\text { ción }\end{array}$ \\
\hline Sensitivo & $\begin{array}{l}\text { Muchos ejercicios. Las } \\
\text { conclusiones son presentadas } \\
\text { antes que el material abstracto } \\
\text { de aprendizaje. Los ejercicios } \\
\text { y pruebas de autoevaluación } \\
\text { sólo después del material de } \\
\text { aprendizaje. }\end{array}$ & \\
\hline Intuitivo & $\begin{array}{l}\text { Pruebas de autoevaluación y } \\
\text { ejercicios son recomendados } \\
\text { para ser presentados antes del } \\
\text { material de aprendizaje. Ejem- } \\
\text { plos son presentados después } \\
\text { del contenido abstracto. }\end{array}$ & $\begin{array}{l}\text { Presentación } \\
\text { de esquemas } \\
\text { entre temas } \\
\text { Ejemplos y } \\
\text { ejercicios }\end{array}$ \\
\hline Secuencial & $\begin{array}{l}\text { Se presenta primero el material } \\
\text { de aprendizaje, luego algu- } \\
\text { nos ejemplos, y después una } \\
\text { prueba de auto evaluación y } \\
\text { algunos ejercicios. Esquemas } \\
\text { son presentados solo antes del } \\
\text { contenido. }\end{array}$ & \\
\hline Global & $\begin{array}{l}\text { Proporcionar esquemas } \\
\text { adicionalmente entre los temas, } \\
\text { presentar una conclusión } \\
\text { directa después del contenido. } \\
\text { Proporcionar un gran número de } \\
\text { ejemplos después del material } \\
\text { de aprendizaje. Ejemplos, ejer- } \\
\text { cicios, y pruebas de autoevalua- } \\
\text { ción al final. }\end{array}$ & \\
\hline
\end{tabular}

Fuente: Kanninen, Essi (2008, p.28).

Finalmente, mediante la ejecución de las etapas iniciales de la investigación, se proponen las accio- 
nes articuladas que se esquematizan en la Figura 4, para lograr un diseńo didáctico adaptado al aprendiz, descrito por los resultados obtenidos después de la aplicación del test de Felder \& Soloman.

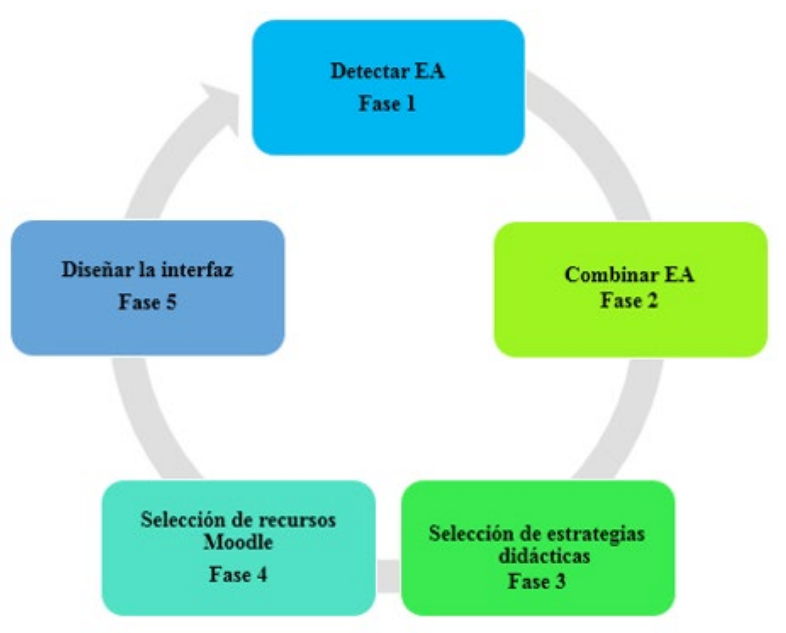

Figura 5.

Etapas del método propuesto.

Elaboración propia (2018).

A continuación, se describe cada una de las fases de la Figura 5, del método propuesto.

Detección de los estilos de aprendizaje de los alumnos: en esta primera fase deberá aplicarse el ILS de Felder \& Soloman. En virtud de que el ambiente eLearning considerado es Moodle, para tales propósitos pudiera considerarse el empleo de la herramienta desarrollada por Puello, Fernández \& Cabarcas (2014), o la modalidad descrita por Gabino \& Salguero (2017), o bien emplear métodos alternativos como los recursos en línea disponibles y/o la publicación del test dentro de la plataforma, tal y como se efectuó a los fines del presente estudio.

Combinación de estilos de aprendizaje: en la segunda fase aplicado el instrumento, caracterizar el modelo de usuario según los estilos de aprender preferidos, tomando en cuenta la combinación de éstos, según lo recomendado por Kanninen (2009), Ramírez \& Rosas, (2014), Cruz Garzón, (2017) y Angel, (2015).

\section{Selección de las estrategias didácticas y soporte} a través de los recursos disponibles en Moodle: en esta tercera y cuarta fase, en virtud de que el esquema de adaptación del diseño didáctico a los efectos de este estudio, se ha enmarcado en la compilación y acoplamiento de los contenidos en la plataforma, se propone la selección de estrategias didácticas tomando en cuenta los estilos de aprendizaje predominantes y la adaptabilidad basada en la colaboración, que puede ser soportada a través de los distintos recursos disponibles y herramientas nativas en un ambiente eLearning basado en Moodle. En cuanto a este tópico, en el trabajo realizado por Angel, (2015), se proporcionan recomendaciones de utilidad con relación a la selección de estrategias didácticas orientadas al trabajo colaborativo y de recursos externos a la plataforma que podrían ser empleados para reforzar la comunicación síncrona y asíncrona, así como favorecer las combinaciones de estilos de aprendizaje.

Diseñar la interfaz según las estrategias y recursos seleccionados: habiendo finalizado la ejecución de las etapas previas (fase 1, 2, 3 y 4) se presenta el esquema de la interfaz adaptada de acuerdo a los criterios ya descritos. En tal sentido, se esgrime la matriz de diseño didáctico que se presenta a continuación, para la combinación de estilos de aprendizaje predominante (Tabla 11). Esta matriz pretende servir de orientación del diseño de la interfaz.

\section{Tabla 11}

Estrategias sugeridas para la adaptación del diseño pedagógico.

\begin{tabular}{|c|c|c|}
\hline Dimensión & Estrategias & Recursos de Moodle \\
\hline Activo & $\begin{array}{l}\text { Presentación del tema } \\
\text { a través de un esque- } \\
\text { ma general o diagrama } \\
\text { de flujo. El contenido } \\
\text { debe contener ilustra- } \\
\text { ciones, apoyadas por } \\
\text { elementos audiovisua- } \\
\text { les enlazados o bien } \\
\text { embebidos dentro de } \\
\text { la plataforma. }\end{array}$ & $\begin{array}{l}\text { Inserción de Foro/Chat } \\
\text { de discusión relativo } \\
\text { a la materia y a los } \\
\text { resultados del caso de } \\
\text { estudio/ simulación. }\end{array}$ \\
\hline Sensitivo & $\begin{array}{l}\text { Planteamiento de } \\
\text { microprácticas y/o } \\
\text { caso de estudio } \\
\text { (situación problemáti- } \\
\text { ca) a resolver a través } \\
\text { de simulaciones y } \\
\text { actividades tipo drag } \\
\text { and drop, después del } \\
\text { contenido. }\end{array}$ & $\begin{array}{l}\text { Colocar como } \\
\text { prerrequisito para rea- } \\
\text { lizar la lección/quiz de } \\
\text { autoevaluación, haber } \\
\text { recorrido los conteni- } \\
\text { dos de aprendizaje y } \\
\text { haber participado en el } \\
\text { chat/foro de debate. El } \\
\text { orden de las preguntas } \\
\text { debe obedecer al del } \\
\text { contenido. }\end{array}$ \\
\hline
\end{tabular}




\begin{tabular}{lll}
\hline Dimensión & \multicolumn{1}{c}{ Estrategias } & \multicolumn{1}{c}{ Recursos de Moodle } \\
\hline Secuencial & $\begin{array}{l}\text { El esquema deberá ser } \\
\text { introducido antes que } \\
\text { el contenido, en un } \\
\text { orden específico. }\end{array}$ & $\begin{array}{l}\text { Autoevaluación a } \\
\text { través de lecciones } \\
\text { o quizes después de } \\
\text { la presentación del } \\
\text { contenido. Se aconseja } \\
\text { la lección diseñada con } \\
\text { Visual }\end{array}$ \\
& $\begin{array}{l}\text { prerrequisitos. } \\
\text { llustraciones de apoyo } \\
\text { para la presentación } \\
\text { de esquemas, con alta } \\
\text { definición. Embeberl } \\
\text { enlazar contenido en } \\
\text { formato multimedial vi- } \\
\text { sual en la presentación } \\
\text { de los contenidos. }\end{array}$ \\
\hline
\end{tabular}

Fuente: Elaboración propia (2018).

De acuerdo a la información mostrada en la Tabla 11 y con el fin de atender a las necesidades de los discentes, se propone un ambiente eLearning con una interfaz amigable, cuyo contenido se ubique dentro del diagrama de temas. Para fijar las unidades de información, las actividades y los recursos de aprendizaje, deberá hacerse uso del bloque respectivo.

La información deberá ser presentada en una secuencia lógica: Esquema/Diagrama de flujo de contenidos- (recursos multimediales-visuales) microprácticas-Chat y/o Foro de debate-Autoevaluación.

Los contenidos deberán estar compuestos por alto contenido de imágenes y situaciones problemáticas sometidas a discusión, recursos multimediales visuales en su presentación y lecciones con prerrequisitos basados en la secuencia didáctica.

\section{Discusión y Conclusiones}

Con la materialización de este estudio, se pudieron identificar los estilos de aprendizaje de los alumnos del programa eLearning Afectivo e Inclusivo: Principios y Estrategias, para la adaptación de un ambiente eLearning -cuya base es el LMS Moodle-, a través de la aplicación del esquema de 5 fases propuesto para generar la adecuación de la didáctica.
Tomando como base la información recolectada a través de la aplicación del instrumento desarrollado por Felder \& Soloman y la aplicación de la escala utilizada por Felder \& Spurlin (2005) y Guanipa \& Mogollón (2006), en esta investigación, se tiene que, a nivel general, el alumnado se orientó hacia el nivel equilibrado en las cuatro dimensiones. Se observa entonces que los alumnos participantes se orientan bilateralmente, sin beneficiar un lado a expensas del otro.

Considerando los niveles, se obtuvo una preferencia por los estilos: global (fuerte y moderado), reflexivo (moderado y fuerte), intuitivo (fuerte, en el moderado se equiparán los estilos sensitivo e intuitivo), verbal (moderado y fuerte). Por otra parte, se observa que la combinación Activo-Sensitivo, Secuencial-Visual, viene a ser la complementaria a la obtenida en forma general; lo cual conduce a recomendar que, en este caso, el diseño didáctico se adapte a las dos tendencias.

A partir de la investigación documental realizada, se coincide con las posturas de autores como Díaz (2016), Renés (2018), Tapias \& Cué (2016), Rivas \& Gértrudix (2016), Arellano \& Geijo (2016), García, Mansilla \& Ceniceros (2016) y Gordón (2016). En tal sentido, se puede inferir que la metodología didáctica es fundamental para lograr aprendizajes significativos y que ésta no implica la enunciación de "recetarios didácticos" sino más bien una aproximación teórico-conceptual sobre estilos de enseñanza, en cuya construcción es muy importante integrar los estilos de aprender de los discentes - por cuanto marcan una diferencia tanto en la manera de enseńar como de aprendery asimismo, las tipologías propias del ambiente eLearning en el que tiene lugar el hecho educativo. En este sentido, se considera que el dilucidar la predominancia de los estilos de aprendizaje en los discentes es primordial para proponer nuevas técnicas de enseñanza y obtener mejores resultados. A partir de esto, se puede concluir que en la medida en que los contextos educativos evolucionan, los instructores deben acoger una variedad de estrategias pedagógicas y tecnologías innovadoras para permitir el aprendizaje.

Habiendo considerado los hallazgos de Kanninen (2009), Pineda, Valdivia \& Ciraso (2019), An- 
telm, Cacheiro-González, \& Gil-López, (2015) y Macías (2015), se pudo demostrar que es posible definir un procedimiento de adaptación del diseño didáctico enmarcado en la detección y montaje de los contenidos, cuya selección se propone que se realice tomando en cuenta el predominio del estilo de aprender -obtenido a partir de la aplicación del test de Felder \& Soloman- y la adaptatividad basada en la colaboración, que según Avello \& Duart (2016), favorecen la interacción social entre los discentes y puede ser soportada a través de los distintos recursos disponibles en un ambiente eLearning basado en Moodle, siguiendo las cuatro fases descritas en los párrafos precedentes.

Al finalizar la redacción del presente artículo, se han encontrado las respuestas ya expresadas. Sin embargo, a partir de las mismas pudieran generarse otras preguntas que podrían ser el tema de futuras investigaciones. Hasta aquí se informó acerca de cómo podría adaptarse un diseño didáctico a estilos de aprendizaje considerando a Moodle como base del ambiente eLearning. En tal sentido, pudieran abordarse nuevas investigaciones tendientes a la aplicación de la metodología propuesta para su posterior evaluación; pues como bien lo señala Vivas (2015), “...las metodologías didácticas no son algo sobre lo que se debe reflexionar e investigar una sola vez", p.88; ya que es una prioridad no limitar los métodos porque hay que experimentar, en función de la pertinencia educativa.

\section{REFERENCIAS BIBLIOGRÁFICAS}

Adams, N. \& Devaney (2009). Measuring conditions conducive to knowledge development in virtual learning environments: Initial development of a model-based survey. The journal of technology, learning, and assessment, 8, 1. Education Resources Information Center, ERIC. Recuperado de http://www.eric.ed.gov/PDFS/EJ859098.pdf

Alonso, Duque \& Correa (2013). Modelos de estilos de aprendizaje: una actualización para su revisión y análisis. Revista Colombiana de Educación. (64).79-105. https://doi.org/10.17227/01203916.64rce79.105

Amaro de Chacín, R. (2011). La planificación didáctica y el diseño instruccional en ambientes virtuales. Investigación y Postgrado, 26(2), 93-128. Recuperado de http://ve.scielo.org/scielo.php?script=sci_arttext\&pi$\mathrm{d}=$ S1316-00872011000200004\&lng=es\&tlng=es.
Angel, W.I. (2015). Los estilos de aprendizaje y el trabajo colaborativo en los ambientes virtuales. Universidad Nacional de Educación a Distancia (UNED). Recuperado de http://e-spacio.uned.es/fez/eserv/tesisuned:Educacion-Wiangel/ANGEL_BENAVIDES_WilmerIsmael_Tesis.pdf

Antelm, A., Cacheiro-González, M., \& Gil-López, A. (2015). Análisis del fracaso escolar desde la perspectiva del alumnado y su relación con el estilo de aprendizaje. Educación y Educadores, 18 (3), 471-489.

https://doi.org/10.5294/edu.2015.18.3.6

Arellano, P. R., \& Geijo, P. M. (2016). Una mirada a los estilos de enseñanza en función de los estilos de aprendizaje. Journal of Learning Styles, 9(18).

Athanasios, T (2007). Description of a Virtual Learning Environment for preliminary schools. (Tesis de maestría), Linnaeus University - Växjö Campus.

Avello, R. \& Duart, J. M. (2016). Nuevas tendencias de aprendizaje colaborativo en eLearning: Claves para su implementación efectiva. Estudios pedagógicos (Valdivia), 42(1), 271-282.

https://doi.org/10.4067/S0718-07052016000100017

Cartelli A., Stansfield M., Connolly T., Athanassios J., Magalhães H. \& Maillet, K. (2008). Towards the development of a new model for best practice and knowledge construction in virtual campuses. Journal of Information Technology Education 7. https://doi.org/10.28945/3191

Cemile F. (2008). A multi - agent adaptative learning system for distance education. Tesis doctoral, Middle East Technical University,

Chard (2011). Building a Virtual Classroom: An education environment for the Internet Generation. (Tesis doctoral), Curtin University.

Chavez, N. (2007). Introducción a la investigación educativa. Maracaibo: Gráfica González.

Cruz-Garzón, J.O. (2017) Ambiente eLearning adaptativo en Moodle basado en estilos de aprendizaje: una contribución al éxito escolar. 2-6. Recuperado de http://www.idep. edu.co/sites/default/files/TICon_2017_paper_36_0.pdf

Despotović \& Zrakić (2012). Providing Adaptivity in Moodle LMS Courses. Educational Technology \& Society, 15 (1), 326-338.

Díaz, M.A. (2016). Estilos de aprendizaje y métodos pedagógicos en educación superior. Universidad Nacional de Educación a Distancia (UNED). Recuperado de http://e-spacio. uned.es/fez/eserv/tesisuned:ED-Pg-Educac-Madiaz/DIAZ_ DIAZ_MARCO_ANTONIO_Tesis.pdf.

Febe, C.R. (2012). Diseño didáctico de un Entorno Virtual de Enseńanza - Aprendizaje para la integración academia industria en la disciplina Ingeniería y Gestión de Software en la Universidad de las Ciencias Informáticas. (Tesis doctoral). Universidad de la Habana.

https://doi.org/10.13140/rg.2.2.35442.50887

Felder, R. (1996). Matters of style, 6(4), 18-23. (A. Prism, Ed.). 
Felder, R. M. \& Spurlin, J. (2005). "Applications, reliability and validity of the index of learning styles", Engineering Education, 21 (1),103-112. Recuperado de http://www4. ncsu.edu/unity/lockers/users/f/felder/public/ILSdir/ILS_ Validation(IJEE).pdf.

Felder, R., \& Silverman, K. L. (1988). Learning styles and teaching styles in engineering education. Engineering Education, 78(7), 674-681.

Gabino R., Salguero, A. (2017). Herramienta para detección de estilos de aprendizaje en estudiantes de educación superior. Revista Tecnológica ESPOL - RTE, 30 (3), 106-121.

Gamero, P. (2014). Didáctica para los estilos de aprendizaje. Revista LEX, 12, (13), 351-368. https://doi.org/10.21503/ lex.v12i13.50

García, M.C., Mansilla, J.M. \& Ceniceros, J.C. (2016). Los estilos de aprendizaje y la autonomía en el universitario. Bragança, Portugal. En L. Miranda, P. Alves y C. Morais (Eds.), VII Congresso Mundial de Estilos de Aprendizagem: livro de Atas (pp. 390-402). http://hdl.handle. net/10198/12934

Gómez, M. (2002). Estudio teórico, desarrollo, implementación y evaluación de un entorno de enseñanza colaborativa con soporte informático. (Tesis doctoral). Universidad Complutense de Madrid.

Gordón, F. D. R. A. (2016). Implicaciones del conocimiento de los estilos de aprendizaje en el ejercicio profesional del docente universitario. Journal of Learning Styles, 9 (18).

Graf, S. \& Tommaso, L. (2007). In-Depth Analysis of the Felder Silverman Learning Style Dimensions. Journal of Research on Technology in Education 40, (1), 79-93.

https://doi.org/10.1080/15391523.2007.10782498

Guanipa, M. \& Mogollón, E. (2006). Estilos de aprendizaje y estrategias cognitivas, Revista Ciencias de la Educación, 1, (27), 11-27. http://servicio.bc.uc.edu.ve/educacion/revista/ volIn27/27-1.pdf

Gutiérrez, M. (2018). Estilos de aprendizaje, estrategias para enseñar. Su relación con el desarrollo emocional y aprender a aprender. Tendencias Pedagógicas, 31, 83-96.

https://doi.org/10.15366/tp2018.31.004

Hernández, R., Fernández, C., \& Baptista, M. D. P. (2014). Metodología de la investigación. México: Mc Graw Hill.

Herrera, M. (2006). Consideraciones para el diseño didáctico de ambientes virtuales de. aprendizaje: una propuesta basada en las funciones cognitivas del aprendizaje. Revista Iberoamericana de Educación, 38(5), 1-19.

http://www.rieoei.org/deloslectores/1326Herrera.pdf

Humanante, P. R., García-Peñalvo, F. J., \& Conde-González, M. Á. (2015). Personal Learning Environments and Online Classrooms: An Experience with University Students. IEEE Revista Iberoamericana de Tecnologías del Aprendizaje (IEEE RITA), 10(1), 26-32.

https://doi.org/10.1109/RITA.2015.2391411

Iglesias, R., Ortiz-Repiso, A. \& Picazo, R. (2006). eLearning personalizado en función de estilos de aprendizaje y utilizando estándares educativos emergentes. Recuperado de http://eprints.ucm.es/9033/1/TC2006-43.pdf

International Centre for Educator's Learning Styles (2018). Recuperado de https://eiclsresearch.wordpress.com/typesof-styles/learning-styles/felder-richard/

Kanninen, E. (2009). Learning styles and eLearning. Tampere: Tampere University of Technology. Master's of Science Thesis. Impreso. Recuperado de https://www.researchgate. net/publication/265245183_eLearning _in_the_City_of_ Tampere.

Kolb, D.A. (1984): Experiential learning: experience as the source of learning and development Englewood Cliffs, NJ: Prentice Hall.

Lee, T. (2011). Rethinking connectedness: An investigation into the Access of teacher profesional learning in regional and remote Western Australia. Tesis doctoral, Curtin University. Recuperado de https://espace.curtin.edu.au/bitstream/handle/20.500.11937/246/160053_Broadley\%20 full.pdf?sequence $=2 \&$ isAllowed $=y$

Macías-Romero, W. D (2015)."Estilos de aprendizaje aplicados a las aulas virtuales de lengua extranjera". Rastros Rostros 17, (31), 53-63.

http://dx.doi.org/10.16925/ra.v17i31.1095

Otzen, T. \& Manterola, C. (2017). Técnicas de Muestreo sobre una Población a Estudio. International Journal of Morphology, 35(1), 227-232.

https://doi.org/10.4067/S0717-95022017000100037

Paramythis, A., \& Loidl-Reisinger, S. (2004). Adaptive Learning Environments and eLearning Standards. Electronic Journal on eLearning, 2, 181-194.

Paramythis, A., \& Loidl-Reisinger, S.(2003). Adaptive learning environments and eLearning standards" .In Second European conference on eLearning. 1, 369-379.

Paredes \& Rodríguez, (2006). The application of learning styles in both individual and collaborative learning. Sixth IEEE International Conference on Advanced Learning Technologies (ICALT'06). IEEE, 1141-1142.

Paredes, P. (2008). Una Propuesta de Incorporación de los Estilos de Aprendizaje a los Modelos de Usuario en Sistemas de Enseńanza Adaptativos. (Tesis Doctoral), Universidad Autónoma de Madrid, Madrid.

Paredes, P., \& Rodríguez, P. (2004). Incorporating learning styles into the user model. (Towarda, Ed.) In Advances in Technology-Based Education Knowledge-Based Society, 774-778.

Pesantez, V. R. (2008). Educación Adaptativa en la Web: Estado del Arte. Recuperado de http://repositorio.utpl.edu. ec/bitstream/123456789/3386/1/378X545.p

Pineda, P., Valdivia, P. \& Ciraso, A. (2019). Actividades de Moodle: Manual de buenas prácticas pedagógicas. Recuperado de https://ddd.uab.cat/pub/estudis/2016/149926/ Moodle_buenas_practicas.pdf

Porras, I. (2017). Redes Sociales, Facebook \& Blog según los Estilos de Aprendizaje en Cursos eLearning. Hamut'ay, 4 


\section{(1), 60-74. https://doi.org/10.21503/hamu.v4i1.1395}

Puello, P. Fernández, D. \& Cabarcas, A. (2014). Herramienta para la Detección de Estilos de Aprendizaje en Estudiantes utilizando la Plataforma Moodle. Formación universitaria, 7(4), 15-24.

https://doi.org/10.4067/S0718-50062014000400003

Ramírez, Y., \& Rosas, D. (2014). Aplicación de la teoría de estilos de aprendizaje al diseńo de contenidos didácticos en entornos virtuales. Eticanet (14).

Renés, P. (2018). Planteamiento de los estilos de enseñanza desde un enfoque cognitivo-constructivista. Tendencias PedagóGicas, 31, 47-68.

https://doi.org/10.15366/tp2018.31.002

Revelo, O., Collazos, C. \& Jiménez, J. (2018). El trabajo colaborativo como estrategia didáctica para la enseñanza/ aprendizaje de la programación: una revisión sistemática de literatura. Revista TecnoLógicas, 21(41), 115-134.

https://doi.org/10.22430/22565337.731

Rivas, B., De Cisneros, J.C. \& Gértrudix, F. (2016). Estilos de aprendizaje y uso de datos en abierto. Estudio de caso con estudiantes de nuevo ingreso en la Facultad de Educación de Toledo. Bragança, Portugal. En L. Miranda, P. Alves y C. Morais (Eds.), VII Congresso Mundial de Estilos de Aprendizagem: livro de Atas (pp. 175-188). Recuperado de http:// hdl.handle.net/10198/12934

Solari-Montenegro, G., Rivera-Iratchet, M. \& Velasco-Mur, A. (2017). Prevalencia de estilos de aprendizaje de los estudiantes de primer semestre de tercero, cuarto y quinto año, y de estilos docentes de la carrera de Cinesiología, Universidad de Antofagasta. Revista Fundación Educación Médica (FEM), 20(2), 57-64.

https://doi.org/10.33588/fem.202.880

Skelton, D. (2007). An investigation into the Learning Environments of Blended Delivery (e - learning and classroom) in a Tertiary Environment. Tesis doctoral, Curtin University.

Tapias, M. G., \& Cué, J. L. G. (2016). Estilos de aprendizaje y diseño de estrategias didácticas desde la perspectiva emocional del alumnado y del profesorado. Journal of Learning Styles, 9 (18).

Vélez Ramos, J. B. (2009). Entorno de Aprendizaje Virtual Adaptativo Soportado por un Modelo de Usuario Integral. Universidad de Girona.

Ventura, A., Moscoloni, N., \& Gagliardi R. P. (2012). Estudio comparativo sobre los estilos de aprendizaje de estudiantes universitarios argentinos de diferentes disciplinas. Psicología desde el Caribe Recuperado de http://google.redalyc. org/articulo.oa?id=21324851003

Vilatuña, F., Guajala, D., Pulamarín, J., \& Ortiz, W. (2012). Sensación y percepción en la construcción del conocimiento. Sophia, Colección de Filosofía de la Educación, (13), 123-149.

Vivas H., J. (2015). La pertinencia de los métodos de enseñanza-aprendizaje desde la teleología de la educación. Sophia, Colección de Filosofía de la Educación, (19), 73-91. https://doi.org/10.17163/soph.n19.2015.03 